Supporting information

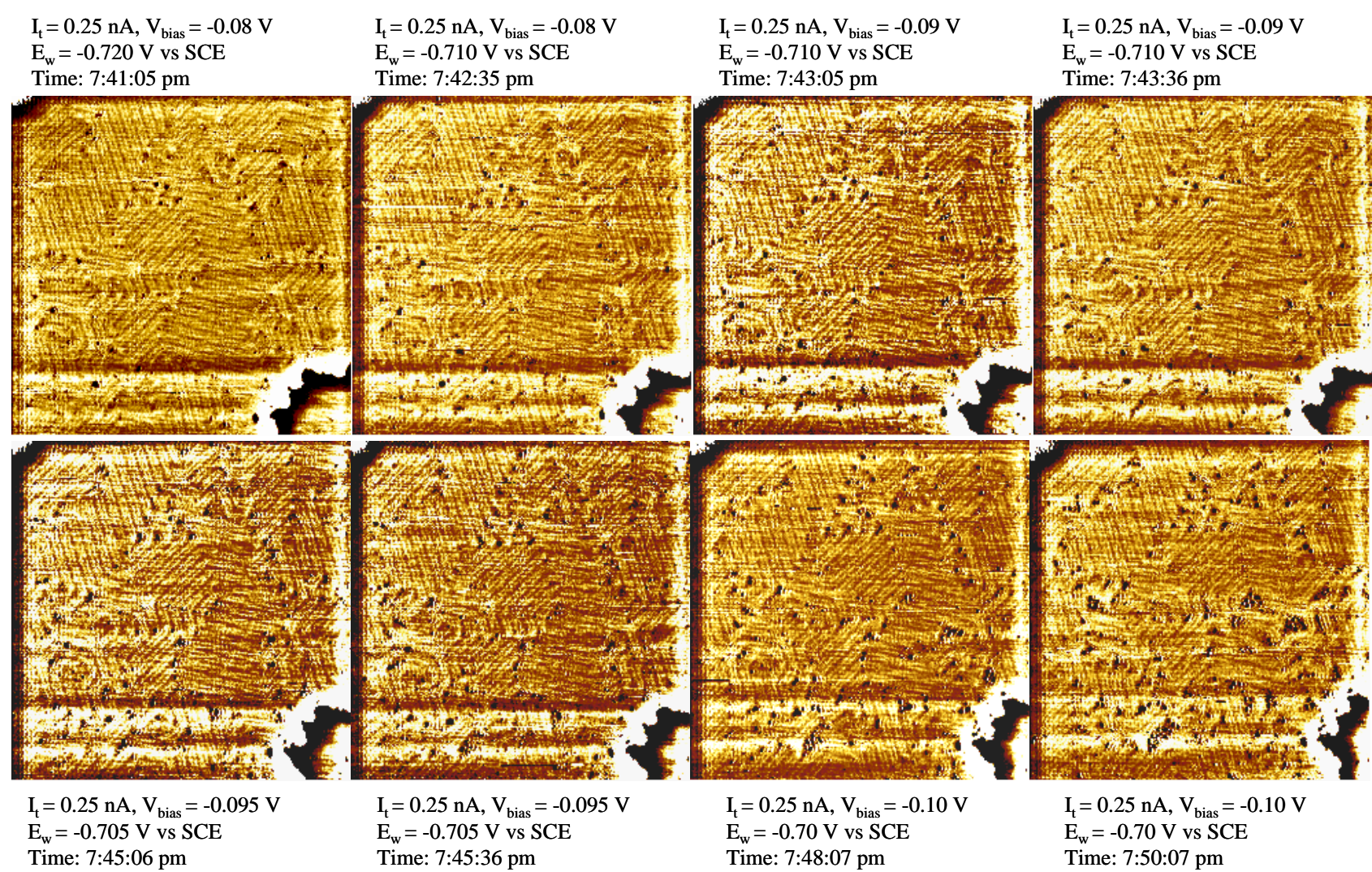




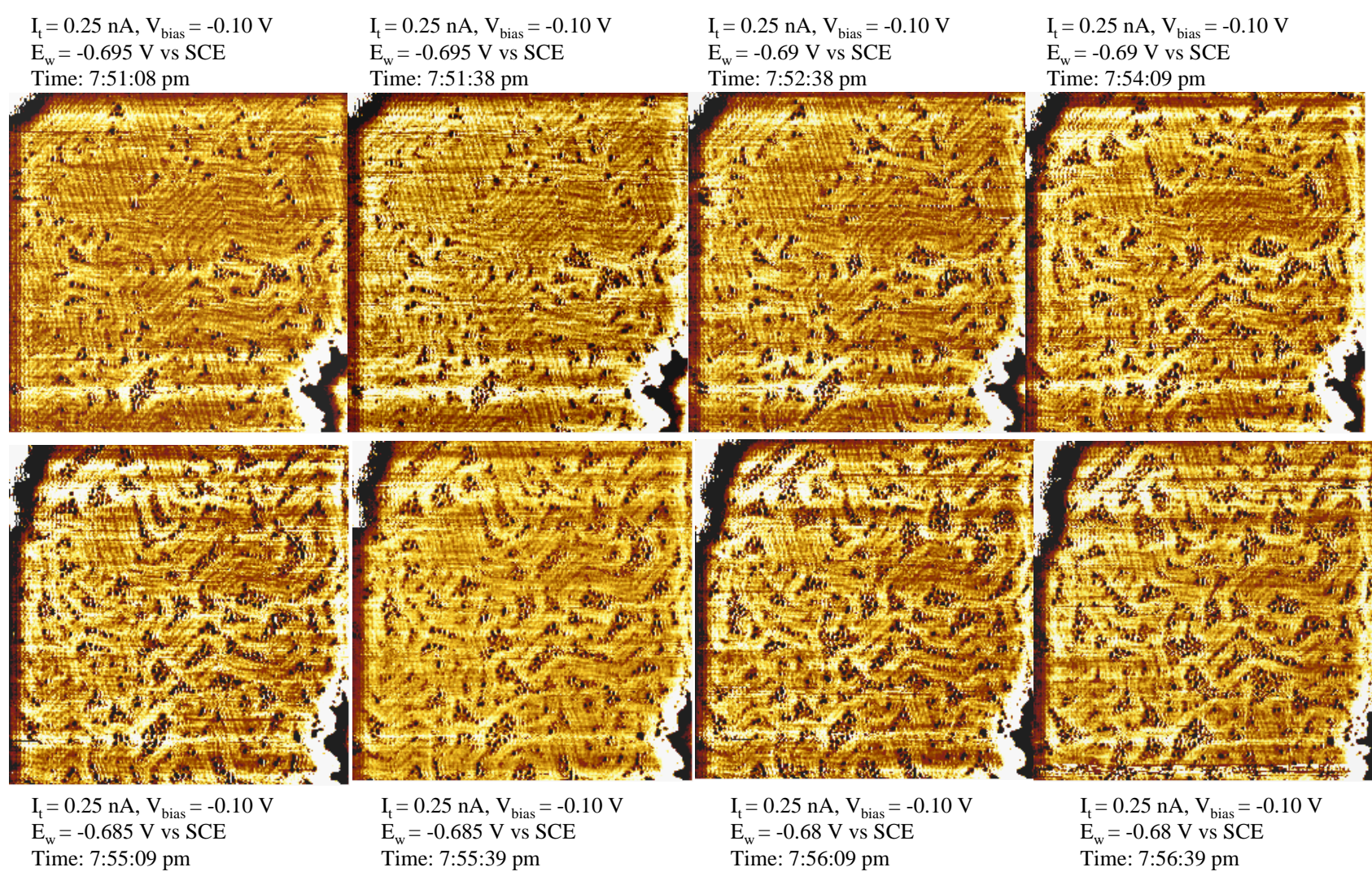


$\mathrm{I}_{\mathrm{t}}=0.25 \mathrm{nA}, \mathrm{V}_{\text {bias }}=-0.10 \mathrm{~V}$ $\mathrm{E}_{\mathrm{w}}=-0.68 \mathrm{~V}$ vs SCE

Time: 7:57:10 pm
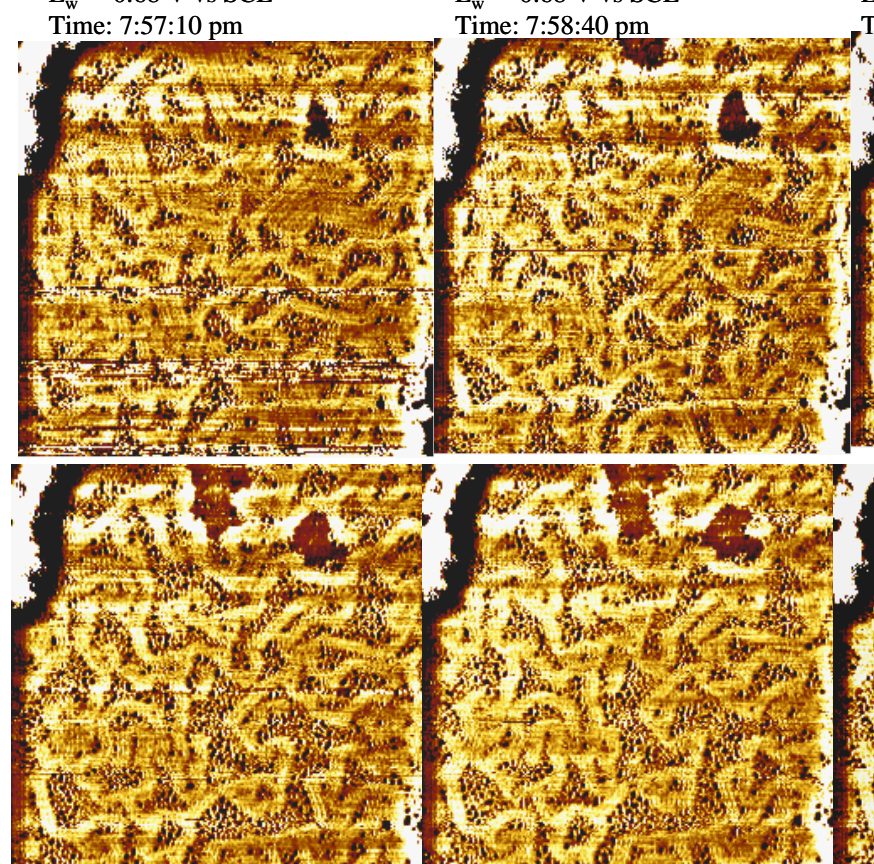

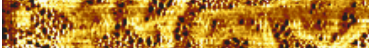

$\mathrm{I}_{\mathrm{t}}=0.25 \mathrm{nA}, \mathrm{V}_{\text {bias }}=-0.10 \mathrm{~V}$

$\mathrm{E}_{\mathrm{w}}=-0.675 \mathrm{~V}$ vs SCE

Time: 8:00:41 pm
$\mathrm{I}_{\mathrm{t}}=0.25 \mathrm{nA}, \mathrm{V}_{\text {bias }}=-0.10 \mathrm{~V}$

$E_{w}=-0.675 \mathrm{~V}$ vs $S C$

Time: 8:01:11 pm
$\mathrm{I}_{\mathrm{t}}=0.25 \mathrm{nA}, \mathrm{V}_{\text {bias }}=-0.10 \mathrm{~V}$

$E_{w}=-0.68 \mathrm{~V}$ vs SCE

The $7.59 .10 \mathrm{pm}$

2. $3 \mathrm{Con}^{3}$

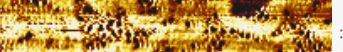

1. $13=8$

$+10$

(2) 30 th

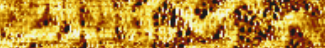

(5) $x^{2}$

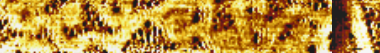

$\mathrm{I}_{\mathrm{t}}=0.25 \mathrm{nA}, \mathrm{V}_{\text {bias }}=-0.10$
$\mathrm{E}_{\mathrm{w}}=-0.68 \mathrm{~V}$ vs SCE 


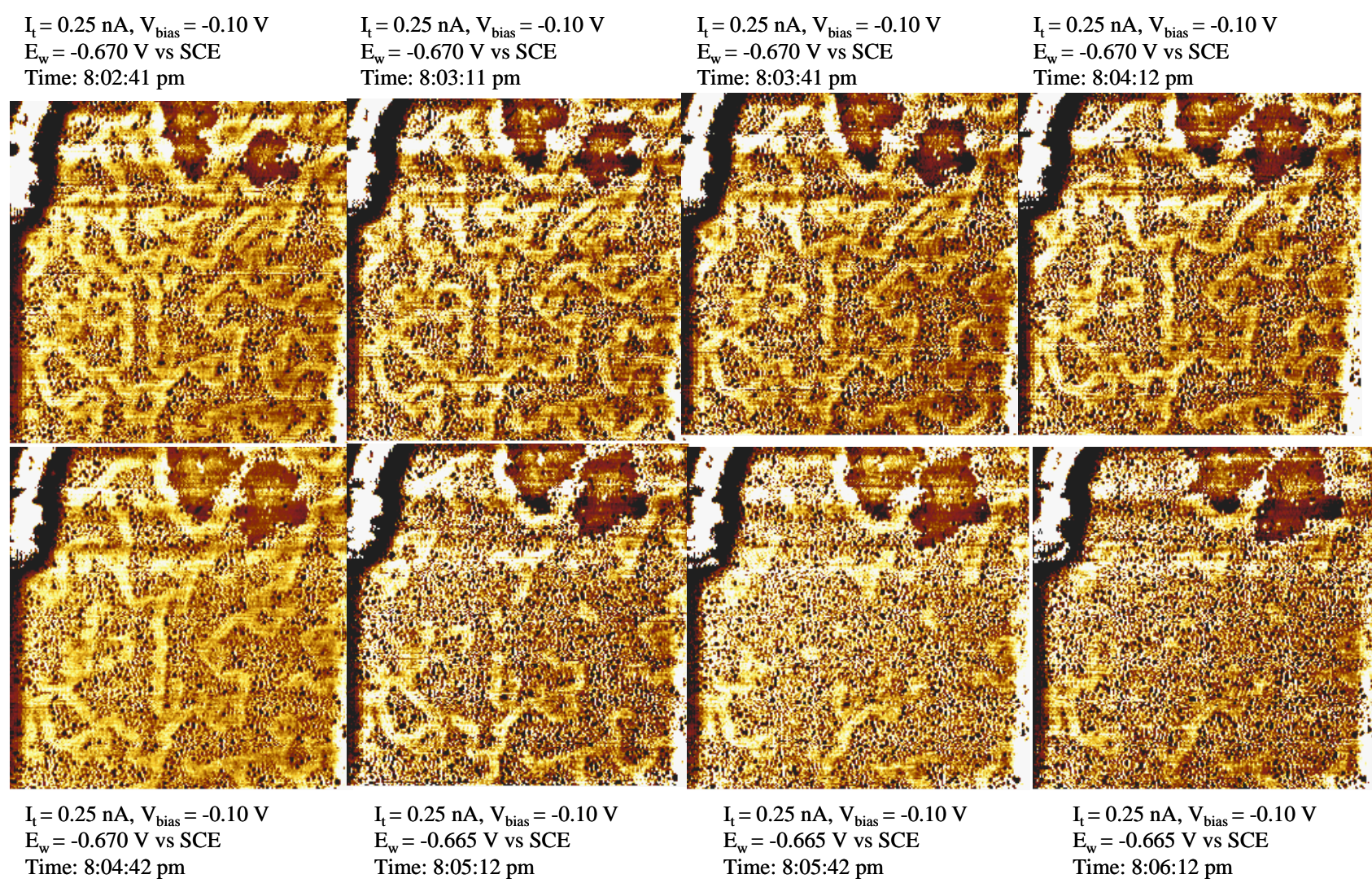




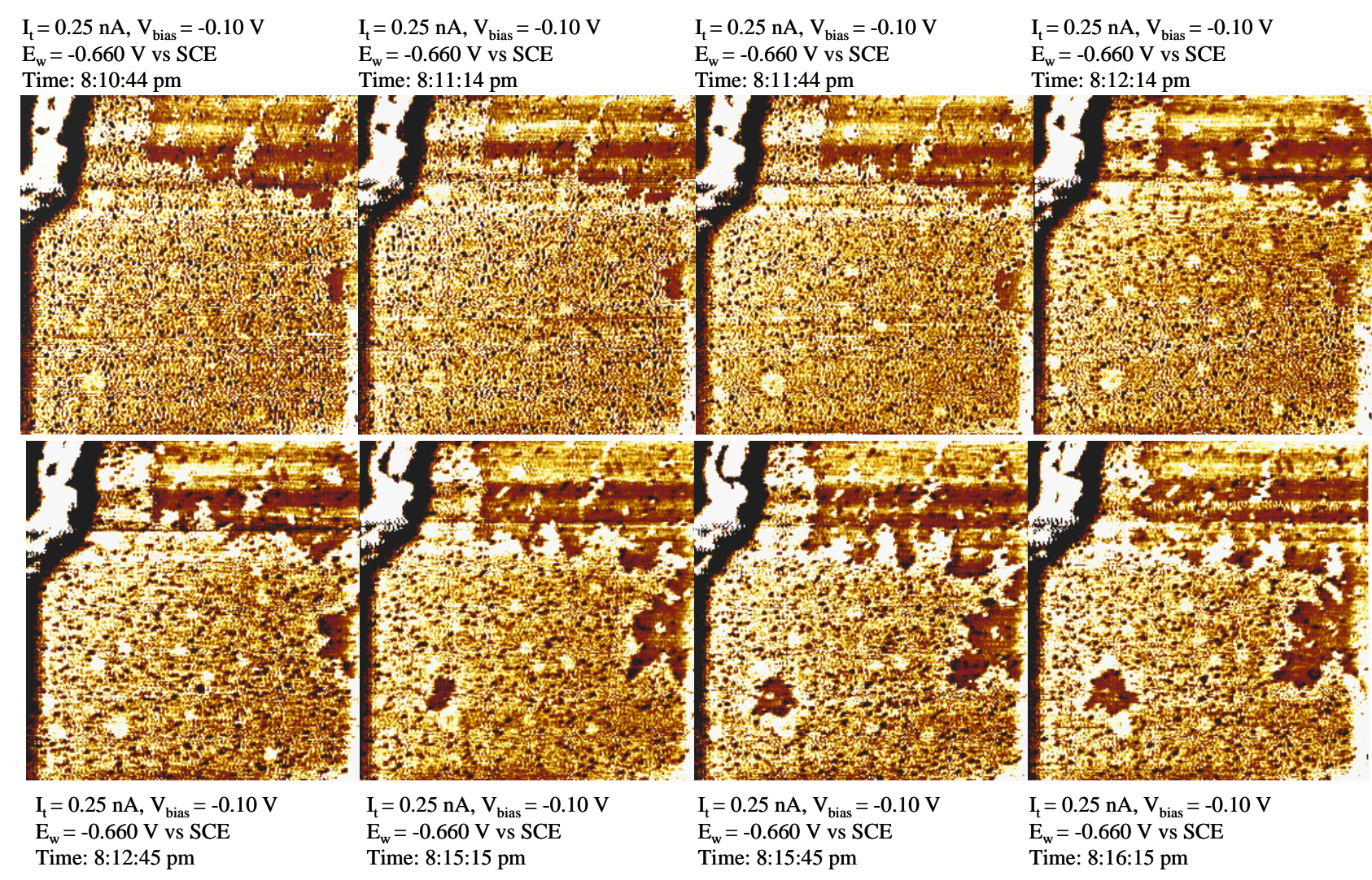




$\begin{array}{llll}\mathrm{I}_{\mathrm{t}}=0.25 \mathrm{nA}, \mathrm{V}_{\text {bias }}=-0.10 \mathrm{~V} & \mathrm{I}_{\mathrm{t}}=0.25 \mathrm{nA}, \mathrm{V}_{\text {bias }}=-0.10 \mathrm{~V} & \mathrm{I}_{\mathrm{t}}=0.25 \mathrm{nA}, \mathrm{V}_{\text {bias }}=-0.10 \mathrm{~V} & \mathrm{I}_{\mathrm{t}}=0.25 \mathrm{nA}, \mathrm{V}_{\text {bias }}=-0.10 \mathrm{~V} \\ \mathrm{E}_{\mathrm{w}}=-0.660 \mathrm{~V} \mathrm{vs} \mathrm{SCE} & \mathrm{E}_{\mathrm{w}}=-0.660 \mathrm{~V} \mathrm{vs} \mathrm{SCE} & \mathrm{E}_{\mathrm{w}}=-0.660 \mathrm{~V} \mathrm{vs} \mathrm{SCE} & \mathrm{E}_{\mathrm{w}}=-0.660 \mathrm{~V} \text { vs SCE } \\ \text { Time: } 8: 16: 45 \mathrm{pm} & \text { Time: } 8: 17: 15 \mathrm{pm} & \text { Time: } 8: 17: 46 \mathrm{pm} & \text { Time: } 8: 18: 16 \mathrm{pm}\end{array}$


$\mathrm{I}_{\mathrm{H}}=0.25 \mathrm{nA}, \mathrm{V}_{\mathrm{brs}}=-0.10 \mathrm{~V}$

$\mathrm{E}_{\mathrm{w}}=-0.660 \mathrm{~V}$ vs SCE

Time: 8:18:46 pm
$\mathrm{I}_{\mathrm{t}}=0.25 \mathrm{nA}, \mathrm{V}_{\text {bias }}=-0.10 \mathrm{~V}$

$\mathrm{E}_{\mathrm{w}}=-0.660 \mathrm{~V}$ vs $\mathrm{S}$
Time: 8:19:16 pm
$\mathrm{I}_{\mathrm{t}}=0.25 \mathrm{nA}, \mathrm{V}_{\text {bias }}=-0.10 \mathrm{~V}$

$\mathrm{t}_{\mathrm{t}}=0.25 \mathrm{nA}, \mathrm{V}_{\text {bias }}=-0.10 \mathrm{E}$
$\mathrm{E}_{\mathrm{w}}=-0.660 \mathrm{~V}$ vs SCE

Time: 8:19:46 pm
$\mathrm{I}_{\mathrm{t}}=0.25 \mathrm{nA}, \mathrm{V}_{\text {bias }}=-0.10 \mathrm{~V}$

$\mathrm{E}_{\mathrm{w}}=-0.660 \mathrm{~V}$ vs SCE

Time: 8:20:16 pm 


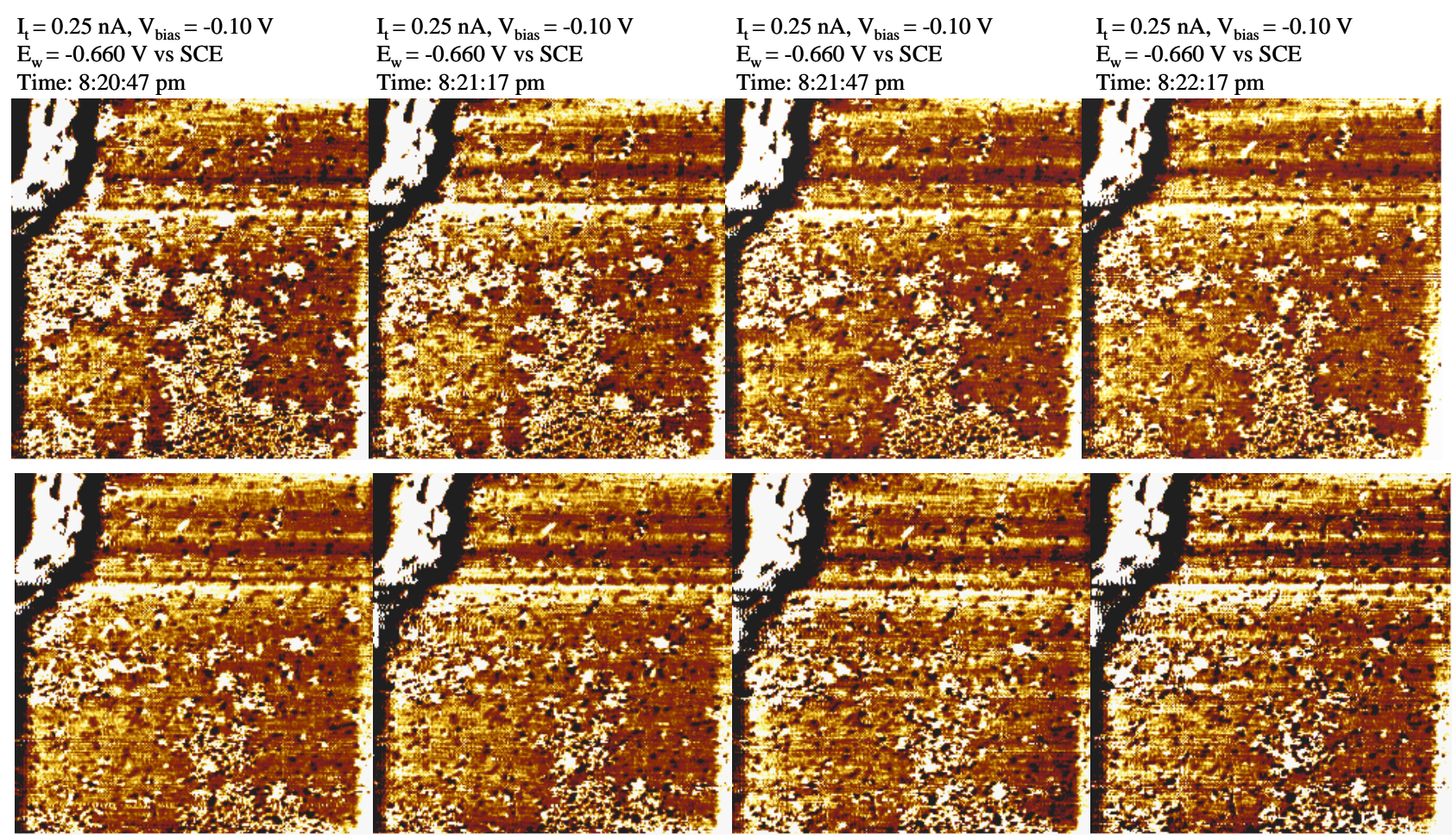
$\mathrm{I}_{\mathrm{t}}=0.25 \mathrm{nA}, \mathrm{V}_{\text {bias }}=-0.10 \mathrm{~V}$
$\mathrm{E}_{\mathrm{w}}=-0.660 \mathrm{~V}$ vs SCE
Time: 8:22:47 pm
$\mathrm{I}_{\mathrm{t}}=0.25 \mathrm{nA}, \mathrm{V}_{\text {bias }}=-0.10 \mathrm{~V}$
$\mathrm{E}_{\mathrm{w}}=-0.660 \mathrm{~V}$ vs SCE
$\mathrm{I}_{\mathrm{t}}=0.25 \mathrm{nA}, \mathrm{V}_{\text {bias }}=-0.10 \mathrm{~V}$
$\mathrm{I}_{\mathrm{t}}=0.25 \mathrm{nA}, \mathrm{V}_{\text {bias }}=-0.10 \mathrm{v}$
$\mathrm{E}_{\mathrm{w}}=-0.660 \mathrm{~V}$ vs SCE
$\mathrm{I}_{\mathrm{t}}=0.25 \mathrm{nA}, \mathrm{V}_{\text {bias }}=-0.10 \mathrm{~V}$
$E_{w}=-0.660 \mathrm{~V}$ vs $S C E$
ime: 8:24:18 pm 


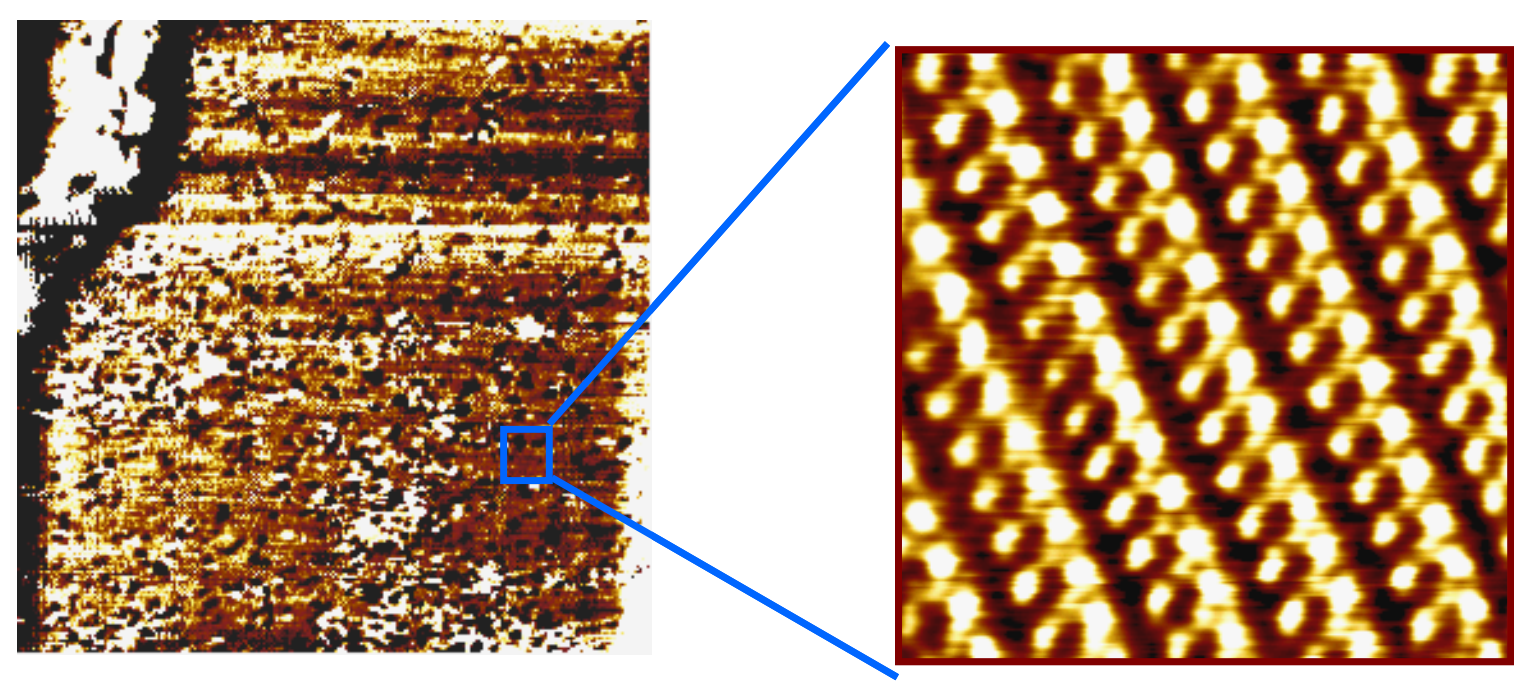

\section{$200 \mathrm{~nm}$}

$$
\begin{aligned}
& \mathrm{I}_{\mathrm{t}}=0.25 \mathrm{nA}, \mathrm{V}_{\text {bias }}=-0.10 \mathrm{~V} \\
& \mathrm{E}_{\mathrm{w}}=-0.660 \mathrm{~V} \text { vs SCE } \\
& \text { Time: } 8: 24: 48 \mathrm{pm}
\end{aligned}
$$

\section{$6 \mathrm{~nm}$}

$$
\begin{aligned}
& I_{t}=0.25 n A, V_{\text {bias }}=-0.10 \mathrm{~V} \\
& E_{w}=-0.660 V \text { vs SCE }
\end{aligned}
$$

Figure s1: Formation of cysteamine monolayer on Au(111) (domain formation) with 65 successive STM images. Scan area $200 \times 200 \mathrm{~nm}^{2}$ except the last image with scan area $6 \times 6 \mathrm{~nm}^{2}$. Other experimental conditions including tunnelling current, bias, potential and time are shown either above or below each image. 


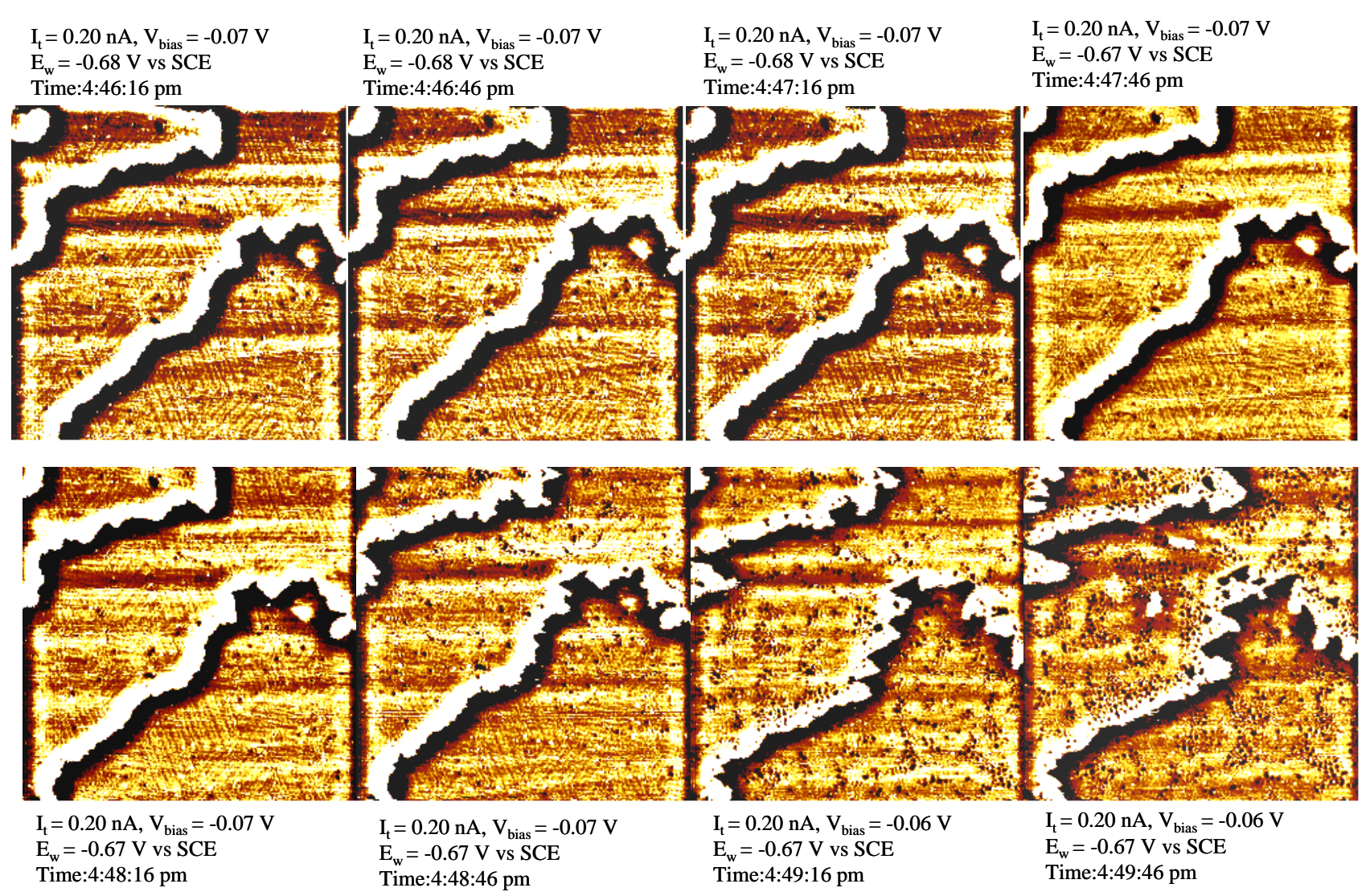




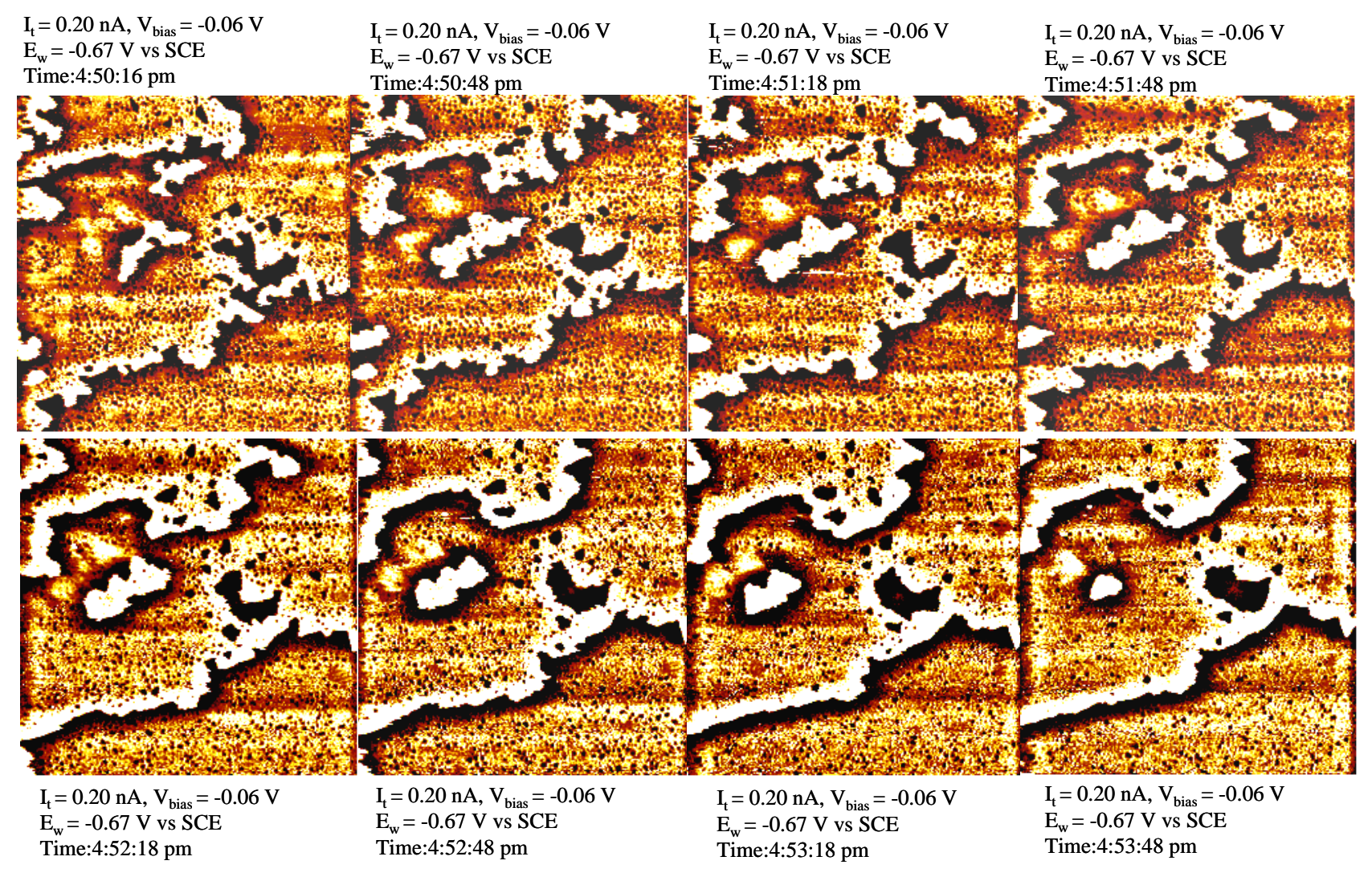

Figure s2: Formation of cysteamine monolayer addressing terrace edges of Au(111) with 16 successive STM images. 